\title{
STERNAL MARROW BIOPSY
}

\author{
By E. Neumark, M.B., B.S.(Lond.) \\ Lecturer in Pathology, St. Mary's Hospital Medical School, London
}

Sternal puncture has become established as a useful diagnostic aid since its introduction by Arinkin (1929) just 20 years ago. The bone marrow in the sternum remains haemopoietically active until late in life and during regeneration shows increased activity more readily than many other bones.

To perform sternal puncture an area of skin at the level of the second or third intercostal space, just to one side of the midline, is anaesthetized and through it the periosteum is infiltrated. The

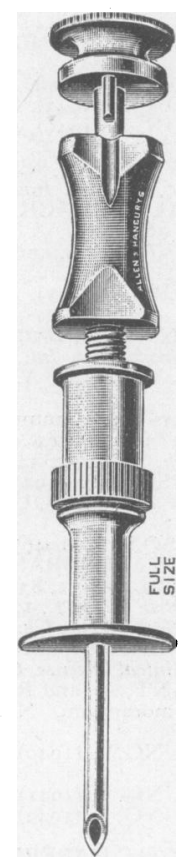

Fig. 1.-Sternal puncture needle. Klima's pattern, modified by Britton.

puncture needle (Fig. I) is pushed through the skin and its guard fixed $0.5 \mathrm{~cm}$. above the skin level. The cortex of the sternum is pierced slightly obliquely by gentle rotatory movements, while the patient holds his breath in inspiration for a few seconds. A sudden give is felt when the spongy bone marrow is reached. 'A well-fitting $5 \mathrm{ml}$. syringe which has been rinsed in saline is attached to the needle and about $0.3 \mathrm{ml}$. of fluid is aspirated. The patient experiences a short, sharp pain whe $\vec{e}$ suction is made. The marrow material is placed in a watch glass and smears are made at once and dried by waving them rapidly. If desired the tot nucleated cell content is estimated by the metho used for counting leucocytes. The remaining specimen is allowed to clot, fixed in Helly's fluiê sectioned and stained like a histological preparatio Macroscopic examination of the material shouf not be neglected. An increase of fat globules may indicate aplastic or hypoplastic conditions, and as increase of little greyish marrow fragments occurs in hyperplasia. The smears are stained with Leishman's or other similar stains and examinead microscopically. Megakaryocytes are usual easily identified with the low power lens, and cont firm that the material obtained is in fact bon $\vec{E}$ marrow. The marrow smears are carefully stumed with the immersion lens and at least 500 cellsciare counted and classified. The normal figures fof sternal marrow counts (Whitby and Britton, 1949 are shown in Table $\mathbf{I}$.

\section{TABLe I}

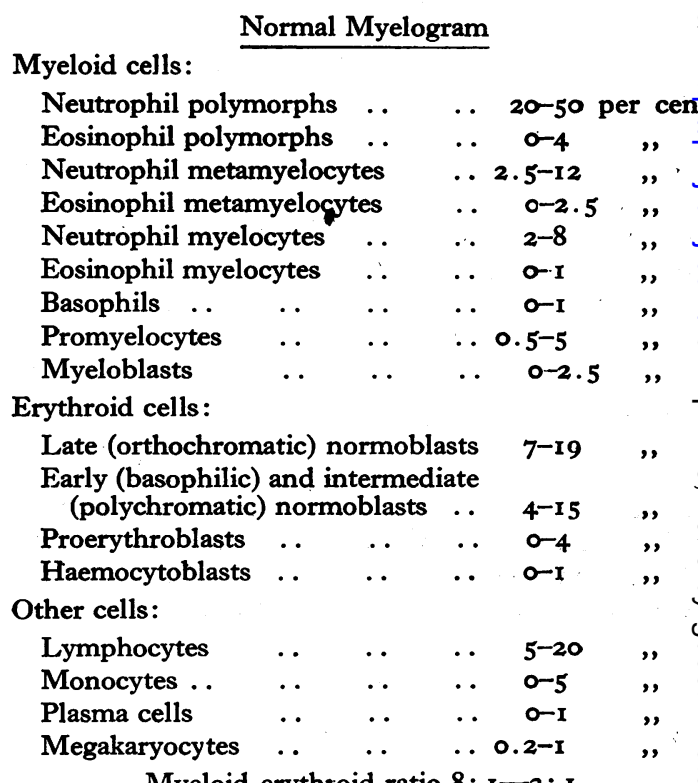
Myeloid-erythroid ratio 8: $1-2: x$ 
TABLE 2

Origin of Bi.ood CelLo

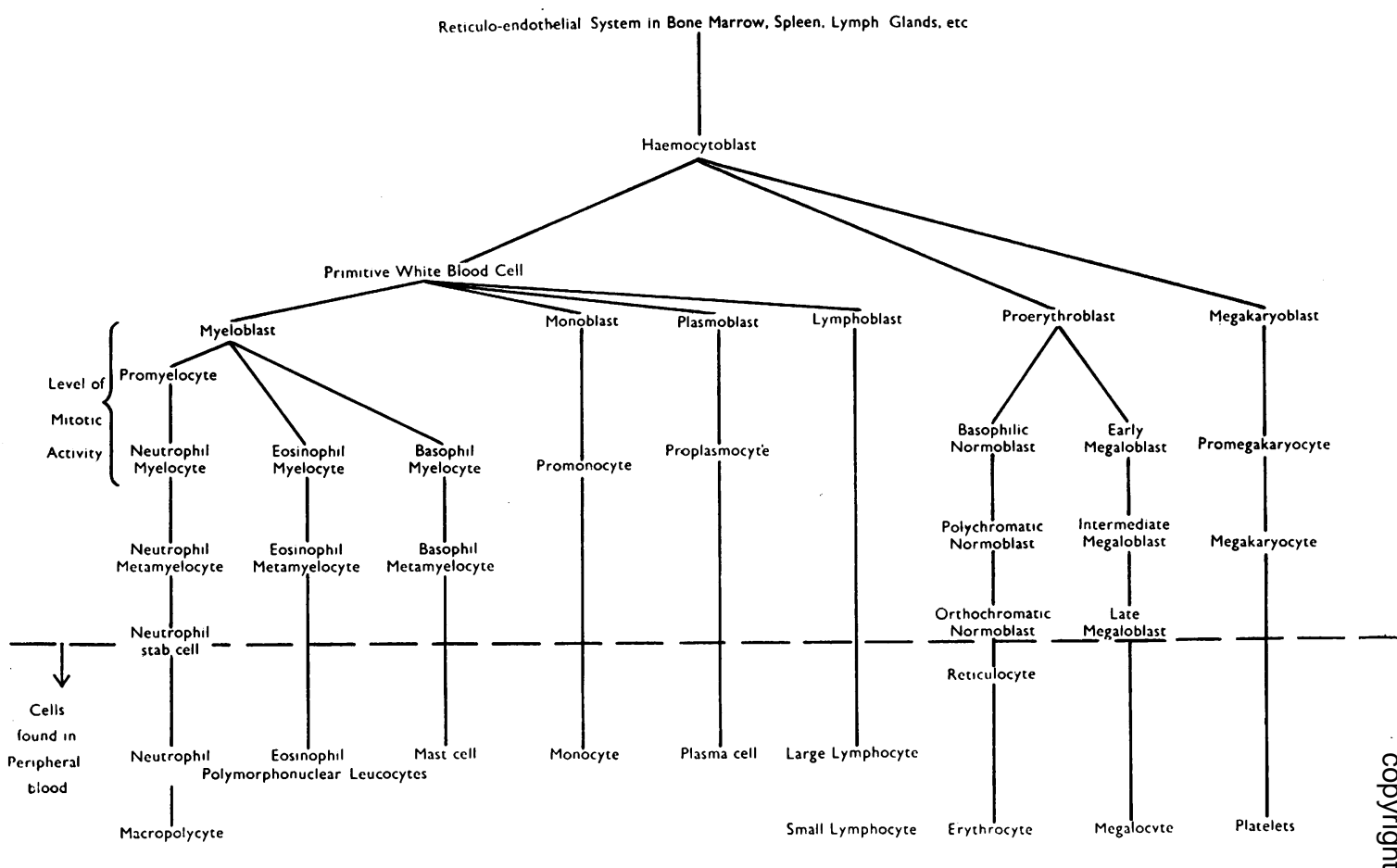

The development of cells is shown diagrammatically in Table 2, but it should be rememb. red that megaloblasts are not normally seen in the marrow, and megalocytes do not normally occur in the blood. Their morphology can be studied in the more detailed books, such as the monograph by Leitner (1949). Mitotic figures can be readily studied in sternal marrow preparations and in many instances provide useful information in assessing disorders of the haemopoietic organs, their activity, severity and prognosis.

In the untreated case of pernicious anaemia the marrow is characterized by megaloblastic hyperplasia, but the myeloid cells and the megakaryocytes also show certain changes, such as large cell forms. Treatment with liver should not be commenced until a definite diagnosis is reached. Following the administration of liver in adequate amounts the megaloblastic marrow becomes transformed into normoblastic marrow, often as rapidly as in 6 to 12 hours. Sprue, pernicious anaemia of pregnancy, tropical megalocytic anaemia and achrestic anaemia show marrow pictures similar to classical pernicious anaemia. Megaloblasts such as described by Turnbull (1936) are only seen in pernicious and allied anaemias (Figs. 2 and 3 ).
Idiopathic hypochromic anaemia, the haemorrhagic anaemias and most secondary anaemias show normoblastic hyperplasia of the erythropoietic marrow portion. Certain other symptomatic anaemias, such as in nephritis, severe infections, benzol poisoning and in malignant disease, may show hypoplasia and in such cases the prognosis is less favourable, but the normoblasts may increase in response to treatment. In aplastic anaemia, myelosclerosis, and similar conditions the total number of marrow cells is much reduced, and therefore the percentage of lymphocytes may be increased, though their absolute figure is not altered.

In the haemolytic anaemias, such as haemolytic icterus, Cooley's anaemia and in toxic haemolytic states, the increase of erythroblasts is particularly marked and extreme marrow hyperplasia may cause skeletal changes. Polycythaemia rubra vera affects all three marrow systems (red, white and megakaryocytic) by hyperplasia, but in erythrocytosis only the normoblasts are increased. Erythraemic myelosis (di Guglielmo's disease) is characterized by enormous pathological erythroblastic hyperplasia and suppression of the myeloid and megakaryocytic series of cells.

In chronic myeloid leukaemia, sternal marrow 


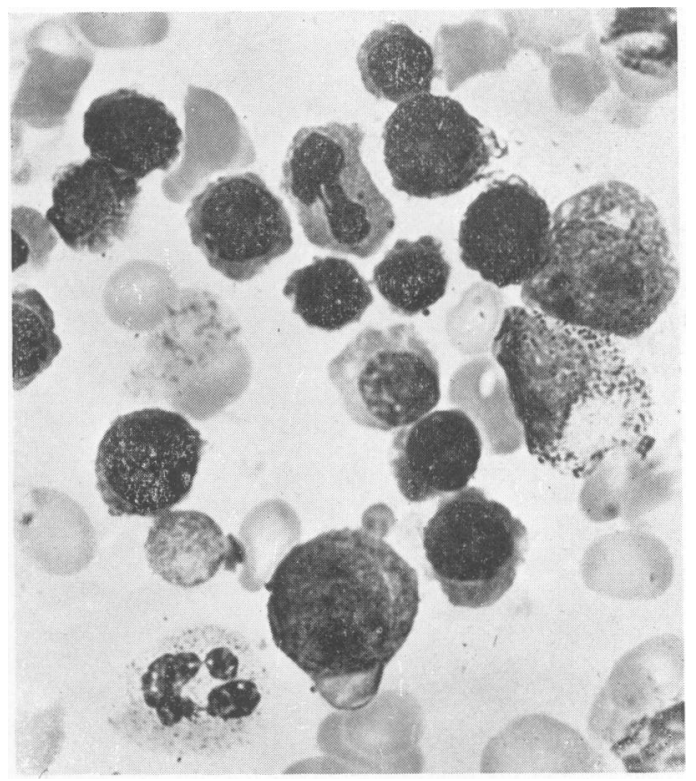

FIG. 2.-Megloblasts, normoblasts and giant myelocytes. The early megaloblast near the polymorph shows the typical fine chromatin structure. From an untreated case of pernicious anaemia (Leishman, x 1,000 ).

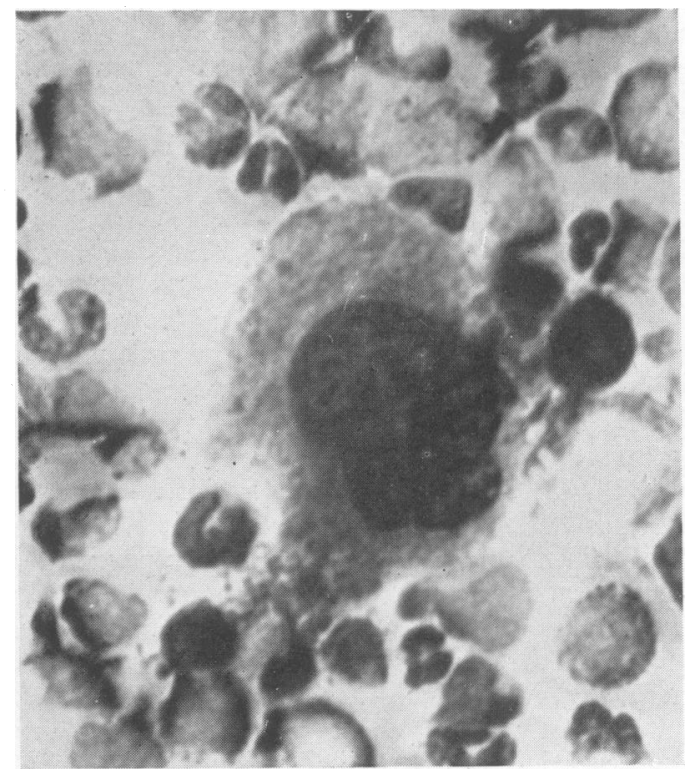

FIG. 4.-Neutrophil polymorphs, myelocytes, promyelocytes and a megakaryocyte. From a case of chronic myeloid leukaemia (Leishman, x I, ০০o).

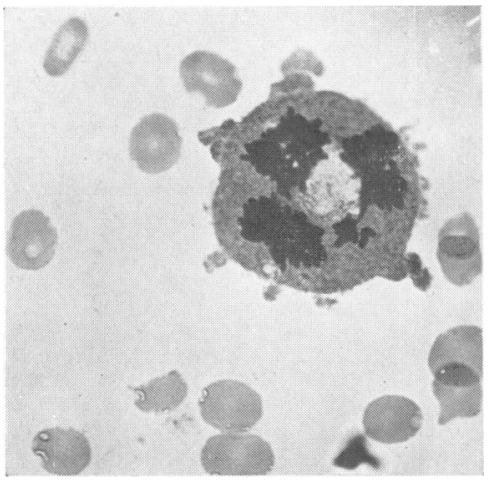

Fig. 3.-An early megaloblast with pseudopodia of the cytoplasm and abnormal mitosis of the nucleus. From a case of pernicious anaemia in relapse (Leishman, x 600).

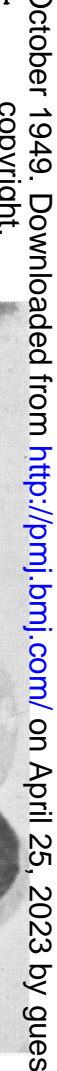

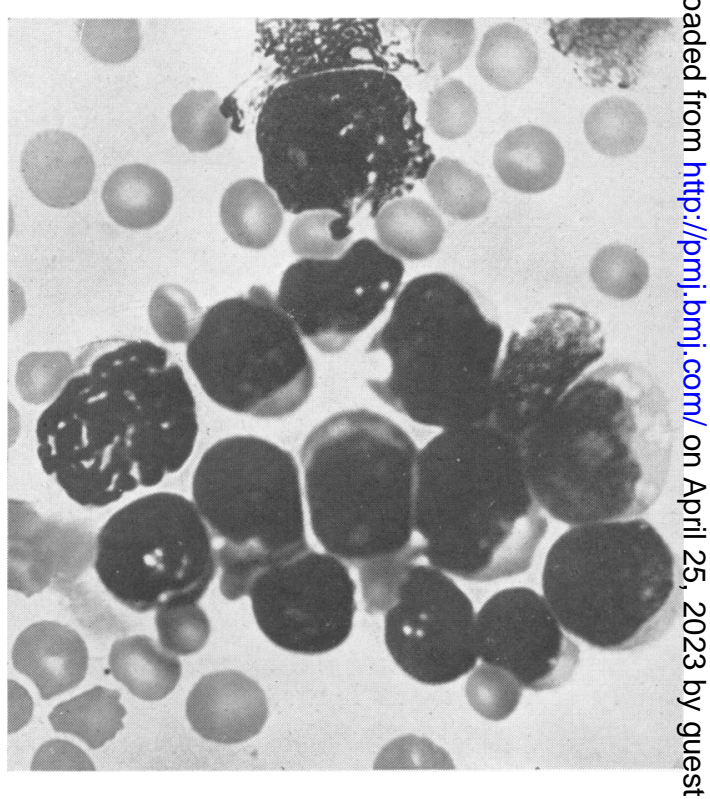

Fig. 5.-Myeloblasts, one of them in mitosis, with littlo cytoplasm and nucleoli in the nucleus. From $\mathbb{8}$ case of acute myeloid leukaemia and chlorom (Leishman, x I, 000). 


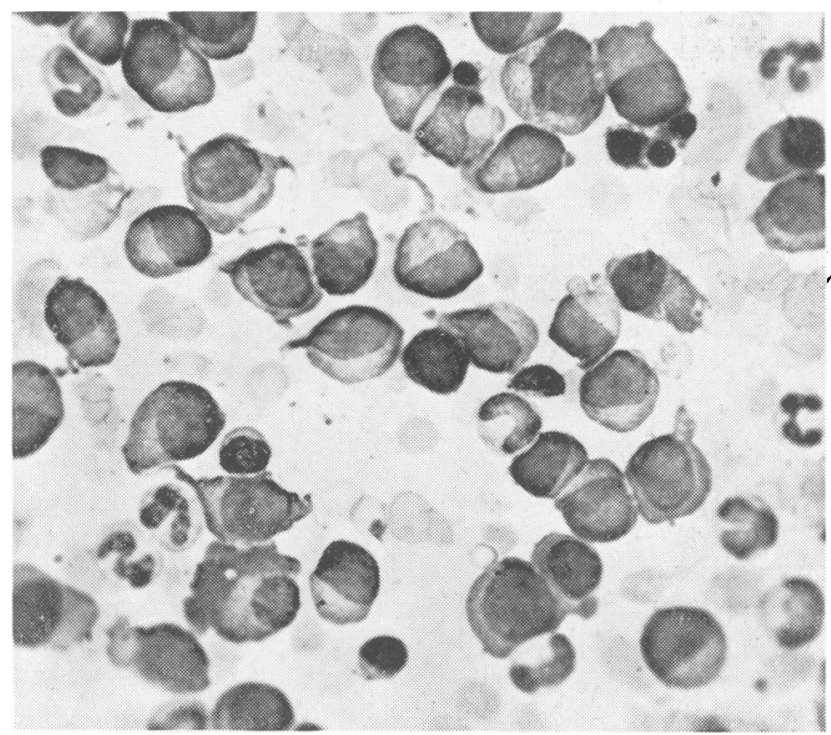

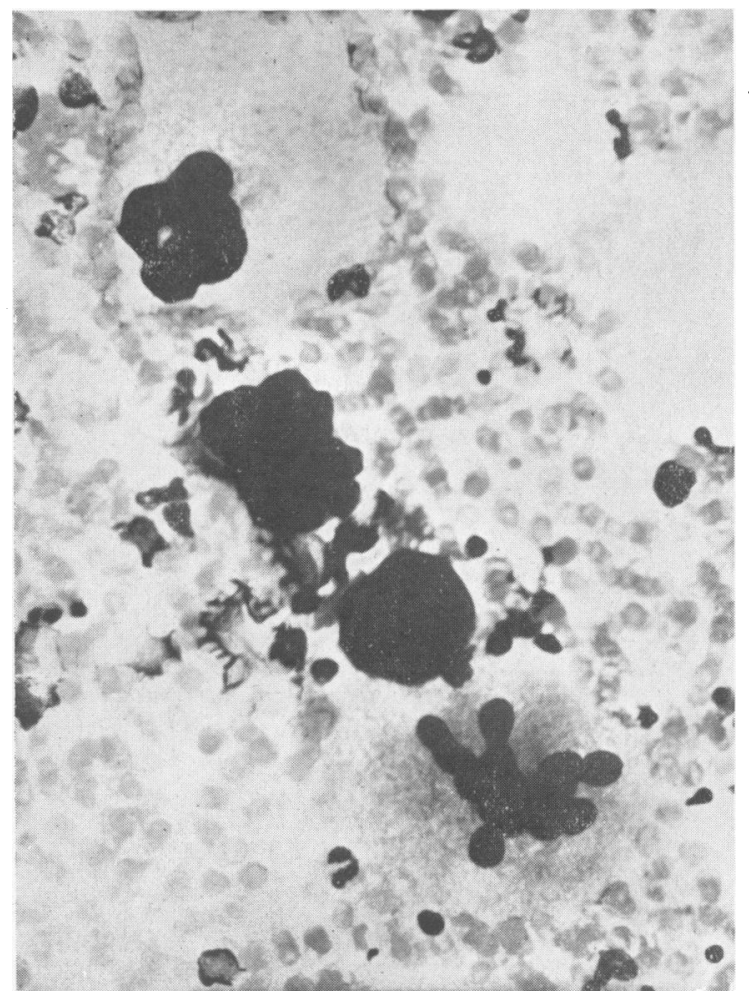

FIG. 7.-Megakaryocytes in various stages of maturation, those with pleomorphic nuclei forming platelets. From a case of idiopathic thrombocytopenic purpura (Leishman, x 500).

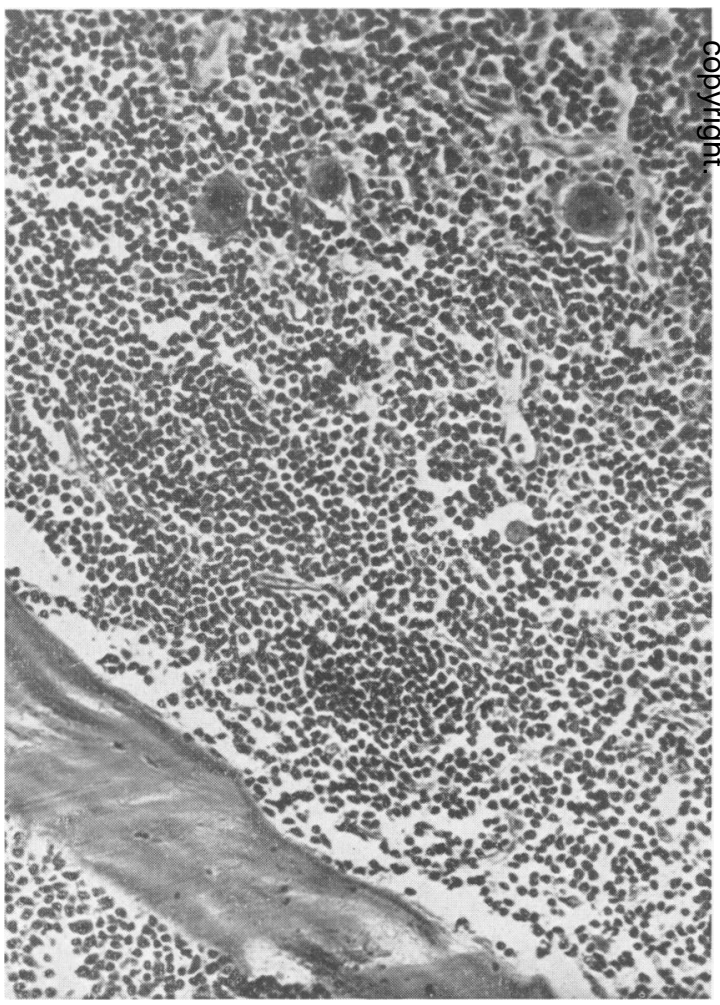

Fig. 8.-Sternal trephine. Infiltration of bone marrow by lymphoid cells while megakaryocytes remain. From a case of lymphatic leukaemia (Haematoxylin and eosin, $x$ 235). 
biopsy produces a picture similar to that found in the peripheral blood, but it is in the cases without leucocytosis that sternal puncture is of diagnosic importance (Figs. 4 and 5). Lymphatic leukaemia does not affect the bone marrow primarily and therefore in early cases a characteristic myelogram cannot always be expected. Once the bone marrow is infiltrated (Fig. 6) the number of lymphocytes and immature lymphoid cells in the marrow is much increased. In acute leukaemia the distinction between myeloid and lymphatic leukaemia is often impossible, even with the help of marrow biopsy ; some cases show myeloblasts with lobed nuclei (' paramyeloblasts' of Naegeli's terminology) which simulate monocytes. In the marrow as well as in the blood these active cells indicate a rapidly progressive disease and herald a fatal prognosis. Mutiple myelomatosis in the majority of cases is easily diagnosed by sternal puncture. The plasma cells or their precursors are unusually numerous, sometimes almost to the exclusion of other cells (Fig. 6).

Sternal marrow biopsy is particularly valuable in the accurate diagnosis and assessment of prognosis in cases of agranulocytosis, aplastic anaemia and other marrow aplasias. In the majority of cases there is maturation arrest with a normal number of early cells and a decrease of polymorphs and late normoblasts only. In such cases recovery may be expected, but when there is aplasia or severe hypoplasia of the myeloid or erythroid series the prognosis must be guarded. Sometimes the true nature of the pathological process can only be revealed by repeated sternal punctures or a sternal trephine.

Some case of glandular fever show a peripheral blood picture which simulates leukaemia. The absence of the atypical cells from the marrow will help when the differential diagnosis is difficult and will exclude leukaemia.

In deciding whether or not a case of thrombocytopenic purpura will benefit from splenectomy sternal puncture is particularly valuable. When the megakaryocytes and their precursors are scanty or absent, splenectomy will rarely be followed by a cure, but when the non-platelet-forming pre megakaryocytes are abundant, splenectomy fre quently results in a removal of the inhibition of maturation (Fig. 7).

Occasionally malignant disease may be diag? nosed by the finding of clusters of cells in the marrow, but these cases are, of course, those wit⿱ skeletal metastases and such advanced stages ma be diagnosed or suspected by the appearance of immature red and white cells in the peripherâ blood, i.e. leuco-erythroblastic anaemia. If Hodgkin's disease, late cases may show the typical mirror-image giant cells, usually associated witto the names of Dorothy Reed and Sternberg. Fogf the detection of malarial parasites, leishmania microfilariae sternal puncture is better than splenie puncture because it is much safer.

Sternal puncture has been used for blood transio fusion, infusions and the injection of therapeutie preparations, but such a procedure is difficult and often hazardous. Intramedullary infusions cag only be given very slowly and they are therefore useless in an emergency.

A thorough general search of marrow smears the differential count of marrow cells in severf stained preparations and the interpretation of the results obtained is a laborious process and usuêllab occupies even a skilled worker for at least hours. It rewards him with a thorough training in cytology and a variety of sometimes very beautitf morphological pictures, especially when mitotio figures are studied.

Fig. 6 is included by courtesy of the Edito St. Mary's Hospital Gazette. The other photo micrographs are by W. Pereira.

\section{BIBLIOGRAPHY}

ARINKIN, M. I. (1929), Folia haemat., Lpz., 38, 233.

LEITNER, S. J. (1949), ' Bone Marrow Biopsy ; Haematology the Light of Sternal Puncture.' English translation, revised are edited by C. J. C. Britton and E. Neumark, J. and A. Churchi\#̈ London.

TURNBULL, H. M. (1936), in Vaughan, J. M. (1936), 'TR Anaemias,' second edition, Oxford Medical Publications London.

WHITBY, L. E. H., and BRITTON, C. J. C. (1946), 'Disorders the Blood,' fifth edition, J. and A. Churchill, London. 